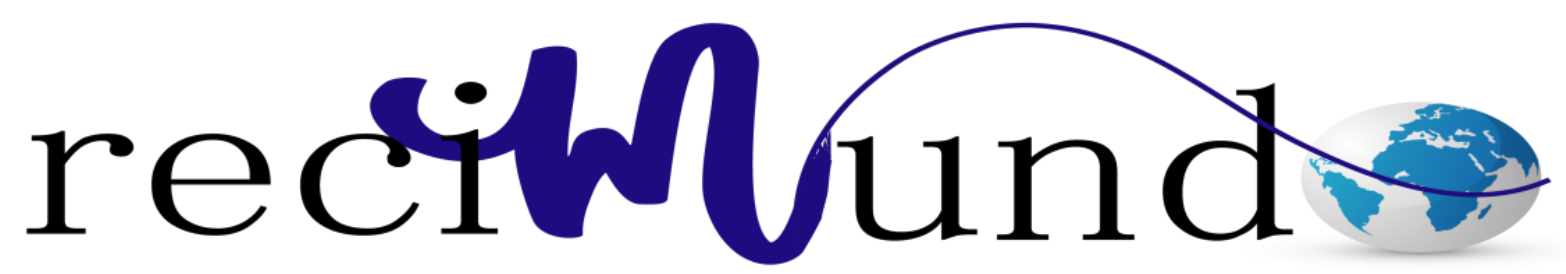

Revista Cientifica Mundo de la Investigación y el Conocimiento

Andrés Alberto Vásquez Gaibor ${ }^{\text {a}}$; Stefanie Cristina Reinoso Tapia ${ }^{\text {b; }}$ María Nora

Lliguichuzca Calle $^{\text {c; }}$ Jim Víctor Cedeño Caballero ${ }^{\mathrm{d}}$

Neumonía asociada a ventilación mecánica

Pneumonia associated with mechanical ventilation

Revista Científica Mundo de la Investigación y el Conocimiento. Vol. 3 núm.3, septiembre, ISSN: 2588-073X, 2019, pp. 1118-1139

DOI: $10.26820 /$ recimundo/3.(3).septiembre.2019.1118-1139

URL: http://recimundo.com/index.php/es/article/view/562

Código UNESCO: 3205 Medicina Interna

Tipo de Investigación: Artículo de Revisión

Editorial Saberes del Conocimiento

Recibido: $15 / 05 / 2019$

Aceptado: 23/06/2019

Publicado: 30/09/2019

Correspondencia: andresvq3@hotmail.com

a. Médico; Investigador Independiente; Guayaquil, Ecuador; andresvq3@ hotmail.com

b. Médico; Investigador Independiente; Guayaquil, Ecuador; stefaniee@ hotmail.es

c. Médico; Investigador Independiente; Guayaquil, Ecuador; noryta2710@ hotmail.com

d. Médico; Investigador Independiente; Guayaquil, Ecuador; doctor@cedenocaballero.com 


\section{Neumonía asociada a ventilación mecánica}

Vol. 3, núm. 3., (2019)

Andrés Alberto Vásquez Gaibor; Stefanie Cristina Reinoso Tapia; María Nora Lliguichuzca

Calle; Jim Víctor Cedeño Caballero

\section{RESUMEN}

La neumonía asociada a la ventilación mecánica [NAVM (NAV)] se encuentra incluida dentro de las infecciones asociadas a la atención sanitaria (IAAS) o también llamadas infecciones "nosocomiales u hospitalarias". Es común en pacientes críticos y es responsable de cerca de la mitad de todos los antibióticos dados a los pacientes en UCIs. El tiempo promedio que se toma desarrollar la NAV desde el inicio de la VM es alrededor de 5 a 7 días, con una tasa de mortalidad referida entre $24 \%$ y $76 \%$. El objetivo de la presente investigación de diseño bibliográfico y, enmarcado en una revisión, es el de desarrollar los aspectos más resaltantes relacionados con la NAVM, entendiéndose éstos como: definiciones de terminología básica, fisiopatología, factores de riesgo, tratamiento y prevención. Los resultados obtenidos logran satisfacer bastamente la meta antes mencionada, puesto que la literatura científico académica disponible es suficientemente extensa y diversa, al igual que vigente. En las conclusiones, entre otros aspectos, se destaca que la traqueostomía con manguito inflado (o el tubo endotraqueal) es considerado uno de los factores de riesgo más destacado en la evolución de esta patología; no obstante, otros estudios aluden a otros trascendentes factores, tales como: la duración de la VM, intubación prolongada o reintubación. La previa exposición a antibióticos, enfermedad pulmonar crónica, bronco aspiración. Por otra parte, se resalta también la relación proporcionalmente directa entre la extensión del tiempo de VM y el riesgo de contagio, que generalmente se deriva en base a las personas que se les intuba para ser auxiliados con VM en un lapso mayor a 48 horas. Finalmente, lo que marca la pauta en el control definitivo de esta patología vendría siendo la alta sospecha clínica el diagnóstico temprano e inicio oportuno del tratamiento antibiótico adecuado a los resultados de las muestras microbiológicas.

Palabras Claves: Infecciones; Traqueostomía; Patología; Intubación; Crónica. 


\section{Neumonía asociada a ventilación mecánica}

Vol. 3, núm. 3., (2019)

Andrés Alberto Vásquez Gaibor; Stefanie Cristina Reinoso Tapia; María Nora Lliguichuzca Calle; Jim Víctor Cedeño Caballero

\section{ABSTRACT}

Pneumonia associated with mechanical ventilation [NAVM (NAV)] is included in infections associated with healthcare (IAAS) or also called "nosocomial or hospital" infections. It is common in critically ill patients and is responsible for about half of all antibiotics given to patients in ICUs. The average time taken to develop the VAP since the beginning of the MV is about 5 to 7 days, with a referred mortality rate between $24 \%$ and $76 \%$. The objective of this research of bibliographic design and, framed in a review, is to develop the most outstanding aspects related to NAVM, these being understood as: definitions of basic terminology, pathophysiology, risk factors, treatment and prevention. The results obtained are enough to satisfy the aforementioned goal, since the available scientific and academic literature is sufficiently extensive and diverse, as well as in force. In the conclusions, among other aspects, it is highlighted that tracheostomy with inflated cuff (or endotracheal tube) is considered one of the most prominent risk factors in the evolution of this pathology; nevertheless, other studies allude to other important factors, such as: the duration of the MV, prolonged intubation or reintubation. Previous exposure to antibiotics, chronic lung disease, bronchoaspiration. On the other hand, the proportionally direct relationship between the extension of the time of $\mathrm{MV}$ and the risk of infection is also highlighted, which is generally derived based on the people who were intuited to be assisted with MV in a period greater than 48 hours. Finally, what sets the standard in the definitive control of this pathology would be the high clinical suspicion the early diagnosis and timely initiation of the antibiotic treatment appropriate to the results of the microbiological samples.

Key Words: Infections; Tracheostomy; Pathology; Intubation; Chronic. 


\section{Neumonía asociada a ventilación mecánica}

Vol. 3, núm. 3., (2019)

Andrés Alberto Vásquez Gaibor; Stefanie Cristina Reinoso Tapia; María Nora Lliguichuzca

Calle; Jim Víctor Cedeño Caballero

\section{Introducción.}

La neumonía asociada a la ventilación mecánica [NAV (NAVM)] se encuentra incluida dentro de lo que la Organización Mundial de la Salud (OMS) (2019 a) menciona como infecciones asociadas a la atención sanitaria (IAAS) o también llamadas infecciones "nosocomiales u hospitalarias", puesto que igualmente se refieren a infecciones que contrae un paciente mientras se le administra su tratamiento en un hospital u otro centro sanitario y que evidentemente este no padecía ni incubaba al momento de su ingreso.

De la misma manera expresan que en cualquier tipo de entorno en el que un paciente reciba la atención sanitaria, puede ser afectado por las IAAS, inclusive, después de haber sido dado de alta.

Las IAAS son el evento adverso más frecuente durante la prestación de atención sanitaria, y ninguna institución ni país puede afirmar que ha resuelto el problema. Según los datos de varios países, se calcula que cada año cientos de millones de pacientes de todo el mundo se ven afectados por IAAS. La carga de IAAS es varias veces superior en los países de ingresos bajos y medianos que en los países de ingresos altos. (OMS, 2019 a)

Este mismo organismo afirma que las causas de la neumonía pueden deberse a virulencias, es decir, a virus, bacterias u hongos. (OMS, 2019 b)

Entonces, la NAV (NAVM) es una de las complicaciones infecciosas que comprenden las neumonías nosocomiales (NN), siendo además considerada como una de las más frecuentes entre los pacientes con insuficiencia respiratoria aguda. (Pérez, Lacarcel, \& López , 2016, p. 525) 


\section{Neumonía asociada a ventilación mecánica}

Vol. 3, núm. 3., (2019)

Andrés Alberto Vásquez Gaibor; Stefanie Cristina Reinoso Tapia; María Nora Lliguichuzca Calle; Jim Víctor Cedeño Caballero

La NAV es común en pacientes críticos y es responsable de cerca de la mitad de todos los antibióticos dados a los pacientes en UCIs. Las cifras dadas por el Consorcio Internacional para el Control de la Infección Nosocomial sugieren que la tasa general de NAV es 13,6 por 1000 días de ventilador. Sin embargo, la tasa individual varía de acuerdo al grupo de pacientes, factores de riesgo y entorno del hospital. El tiempo promedio que se toma desarrollar la NAV desde el inicio de la VM es alrededor de 5 a 7 días, con una tasa de mortalidad referida entre $24 \%$ y $76 \%$. (Miller, 2018)

En el Ecuador la modalidad de vigilancia de esta enfermedad es agrupada. [...] En el 2019 hasta la SE 36 se han reportado 95.431 casos de neumonía, la provincia de Pichinchan notifica el mayor número con 28.345 casos de los notificados a nivel nacional. En la SE 36 se notifican 1.747, siendo la provincia de Pichincha quien notifica el mayor número con 506 casos, seguido de Guayas con 278 casos. El grupo de edad más afectado es 1 a 4 años. (Ministerio de Salud Pública del Ecuador, 2019)

A continuación, se procede a desarrollar los aspectos más resaltantes relacionados con la neumonía asociada a la ventilación mecánica [NAV (NAVM)], entendiéndose éstos como: definiciones de terminología básica, fisiopatología, factores de riesgo, tratamiento y prevención.

\section{Materiales y Métodos.}

El presente material se concibe como el resultado de una revisión a la literatura científico académica reciente y disponible mediante el uso de diversas fuentes bibliográficas encontradas por medio del uso de distintas bases de datos, entre las que figuran: NCBI, SciELO, MedlinePlus, BVS, Embase, PubMed, Dialnet, entre otras. 


\section{Neumonía asociada a ventilación mecánica}

Vol. 3, núm. 3., (2019)

Andrés Alberto Vásquez Gaibor; Stefanie Cristina Reinoso Tapia; María Nora Lliguichuzca Calle; Jim Víctor Cedeño Caballero

Básicamente, la búsqueda se inició poco antes de mediados de septiembre de 2019, utilizando expresiones tales como "neumonía causada por ventilación mecánica", "infección asociada a la ventilación mecánica” y “complicaciones de la ventilación mecánica”, a fines de referir y exponer primordialmente, en base fuentes recientes, qué es y por qué se origina la neumonía a raíz del uso de la ventilación mecánica, rescatando para ello distintos clases de materiales bibliográficos determinados como: artículos científicos, guías clínicas, e-books, ensayos clínicos, consensos, protocolos, revisiones sistemáticas con o sin metaanálisis, estudios de cohorte, casos y controles, series y reportes de casos, boletines y/o folletos de instituciones privadas, formales u oficiales, tesis de posgrado y doctorado, noticias científicas y demás documentos e informaciones que, a criterio del equipo investigador, se caracterice por contener información de interés y de considerable valor en cuanto a la evidencia científica expuesta, que hayan sido publicados, preferiblemente, en los últimos 10 años (incluyendo el presente), es decir, desde 2009 hasta el 2019, haciendo algunas salvedades..

Se desestimaron editoriales, cartas al editor y otros tipos de materiales bibliográficos que igualmente, a juicio de este equipo, se determinaron como de escaso valor cientificoacadémico o con un escaso nivel de evidencia en sus contenidos.

En concreto, cabe destacar que el resultado obtenido tras la búsqueda en dichas bases de datos, remitió a miles de referencias bibliográficas, lo que hizo posible que se comprobara la existencia de suficiente contenido desarrollado en base a esta temática. No obstante, fue ineludible efectuar una selección que redujera significativamente dichos resultados, en función de los objetivos previamente planteados, por lo que se aplicaron, fundamentalmente, criterios de selección tales como: idioma español e inglés, relevancia, correlación temática y fecha de 


\section{Neumonía asociada a ventilación mecánica}

Vol. 3, núm. 3., (2019)

Andrés Alberto Vásquez Gaibor; Stefanie Cristina Reinoso Tapia; María Nora Lliguichuzca Calle; Jim Víctor Cedeño Caballero

publicación en el lapso antes mencionado, en la mayoría de las bases de datos utilizadas.

Paralelamente, se adelantó una búsqueda sin limitación cronológica ni de nivel de evidencia para identificar información igualmente relevante con el término "neumonía" teniendo presente que, a pesar de que éste dista del enfoque primordial, de cierta manera, se considera una búsqueda de información igualmente trascendental para el desarrollo de la presente temática.

Es entonces que, luego de dicho proceso que el equipo investigador le da la correspondiente lectura crítica y análisis interpretativo a toda la evidencia que fue seleccionada, en definitiva, y que sucesivamente derivó en el fundamento de las ideas aquí expresadas de manera consensuada.

La inclusión o exclusión de cada una de las referencias en la presente revisión se definió igualmente por el consenso todos los participantes en este proceso investigativo, y de la misma manera fueron resueltas las decisiones disímiles.

\section{Resultados.}

La OMS define la neumonía como un tipo de infección respiratoria aguda que produce un efecto nocivo en los alvéolos (pequeños sacos) de los pulmones, que en vez de llenarse de aire (lo que sucede en condiciones de salud) lo hacen de líquido y pus, condición que genera que el infectado sienta dolor al respirar y limite su absorción de oxígeno. (OMS, 2019 b)

Para la Sociedad Española de Medicina Interna (SEMI), se entiende por neumonía o pulmonía cuando se produce una inflamación de los espacios alveolares de los pulmones. Típicamente, esta complicación propicia el enrojecimiento, hinchazón y dolencia en el tejido que 


\section{Neumonía asociada a ventilación mecánica}

Vol. 3, núm. 3., (2019)

Andrés Alberto Vásquez Gaibor; Stefanie Cristina Reinoso Tapia; María Nora Lliguichuzca Calle; Jim Víctor Cedeño Caballero

forma los pulmones. Por lo general, esta enfermedad del aparato respiratorio tiene un origen infeccioso, más no debe entenderse como una característica indispensable. Dependiendo de la zona afectada, se puede referir a neumonía lobular (cuando implica por completo un lóbulo pulmonar); bronconeumonía (cuando lo afectado es un segmento de lóbulo, a los alvéolos próximos a los bronquios) o neumonía intersticial (afección asociada al tejido intersticial).

La neumonía adquirida en la comunidad (NAC) o neumonía extrahospitalaria es la que se adquiere fuera de los hospitales, mientras que la neumonía nosocomial (NN) es la que se adquiere durante la estancia hospitalaria, una vez transcurridas las 48 horas o dos semanas después de recibir el alta. (SEMI, 2019)

Ahora bien, en base a Miller (2018)

La NAV es un tipo de neumonía adquirida en el hospital que ocurre más de 48 horas después de la intubación endotraqueal. Puede ser adicionalmente clasificada como de aparición temprana (dentro de las primeras 96 horas de VM) y de aparición tardía (más de 96 horas después de iniciada la VM), la cual es más comúnmente atribuible a patógenos resistentes a múltiples drogas.

Por otra parte, es importante definir de que se trata la ventilación mecánica, para se considera idóneo referir lo establecido por Sociedad Torácica Americana (ATS, por sus siglas en inglés) (2017), de quienes se ha comprendido que esto se trata de un tratamiento de soporte vital con una máquina que auxilia en la función respiratoria de aquellas personas cuando por sí mismas no pueden respirar lo suficiente. 


\section{Neumonía asociada a ventilación mecánica}

Vol. 3, núm. 3., (2019)

Andrés Alberto Vásquez Gaibor; Stefanie Cristina Reinoso Tapia; María Nora Lliguichuzca Calle; Jim Víctor Cedeño Caballero

Así mismo explican que este equipo también suele denominársele ventilador, respirador o máquina de respiración y que, con frecuencia, es en la unidad de cuidados intensivos (UCI) de un hospital donde se atienden a esos pacientes que necesitan apoyo de un ventilador por cuanto padecen de una grave enfermedad. Sin embargo, hacen la salvedad respecto de aquellas personas que requieren de un equipo como éste durante un tiempo prolongado, pues bien pueden aprovecharlo en una unidad regular de un hospital, en un centro de rehabilitación o en el hogar.

Igualmente es necesario esclarecer otros aspectos relacionados con la ventilación mecánica, y para ello, la obra de Vallejo et al. (2019) sirve. Estos expertos, aparte de coincidir con una de las ideas expuestas previamente, al referir que la ventilación mecánica es una de las terapias más usadas en UCI (Unidad de Cuidados Intensivos), resaltan el contraste entre las evidentes ventajas de esta terapia con las complicaciones con las que estrechamente se le vincula. Unas de ellas son la neumonía y la dependencia de la ventilación artificial, ambas concebidas como peligrosas para el paciente que se encuentra en la UCI.

Además, al apoyarse en (Puga et al., 2014) amplían el rango de complejidades ya que éste, respecto al mal manejo del equipo de ventilación mecánica, ha afirmado que "[...] no solo puede producir al paciente consecuencias graves sobre su sistema respiratorio, sino que también pueden ocasionarle graves trastornos hemodinámicos y como consecuencia de ello afectarse todo el organismo" (p. 1141-1142)

De la misma obra se logra extraer que, en base a (Schwonke, Lunardi, \& Silva, 2014), básicamente existen dos tipos de ventilación mecánica: la invasiva y la no invasiva; y aunque ambas compartan la característica de que sus ventilaciones son posibles mediante presión 


\section{Neumonía asociada a ventilación mecánica}

Vol. 3, núm. 3., (2019)

Andrés Alberto Vásquez Gaibor; Stefanie Cristina Reinoso Tapia; María Nora Lliguichuzca Calle; Jim Víctor Cedeño Caballero

positiva, su diferenciación se fundamente en el modo en que esa presión es liberada. De éstos últimos citan que "En la primera se utiliza un tubo oro o nasotraqueal o una cánula de traqueotomía; en la VM [ventilación mecánica] no invasiva el dispositivo conexión entre el paciente y el ventilador artificial consiste en una máscara”. (p. 1143)

Entonces, es así como se encuentran definiciones como las de Vallejo et al. (2019) quienes se atreven a decir que:

La ventilación mecánica invasiva se define como un mecanismo agresivo para paciente pero que a su vez representa una serie de ventajas sobre todo en aquellos casos donde se estima que el mismo estará conectado a ventilación mecánica por un largo periodo de tiempo. Se refiere básicamente al tipo de mecanismo usado para ventilar al paciente, el cual puede ser por medio de un tubo introducido por la vía oro o nasotraqueal o una cánula de traqueotomía. Ventilación mecánica no invasiva. Existe un mecanismo de ventilación menos agresivo, y por tanto con menos complicaciones, que brinda soporte para la respiración por medio de máscaras. (p. 1143-1144)

\section{Fisiopatología}

Aunque clásicamente se han venido distinguiendo 4 vías patogénicas para el desarrollo de NAV (aspiración de secreciones colonizadas procedente de la orofaringe, por contigüidad, por vía hematógena, y a través de los circuitos o tubuladuras), la aspiración de secreciones procedentes de la orofaringe es la vía mayoritaria y casi única. La vía aérea inferior es una zona habitualmente estéril en personas sanas, la excepción se limita a pacientes con enfermedades crónicas pulmonares. En los pacientes bajo 


\section{Neumonía asociada a ventilación mecánica}

Vol. 3, núm. 3., (2019)

Andrés Alberto Vásquez Gaibor; Stefanie Cristina Reinoso Tapia; María Nora Lliguichuzca Calle; Jim Víctor Cedeño Caballero

ventilación mecánica, la intubación endotraqueal, en cambio, rompe el aislamiento de la vía aérea inferior. El neumotaponamiento del tubo endotraqueal es un sistema diseñado para aislar la vía aérea, evitando pérdidas aéreas y la entrada de material a los pulmones, pero no es completamente estanco. Por encima del neumotaponamiento se van acumulando secreciones que, provenientes de la cavidad oral, están contaminadas por los patógenos que colonizan la orofaringe. Estas secreciones contaminadas pasan alrededor del neumotaponamiento y alcanzan la vía aérea inferior. Esta cantidad o inóculo será escaso si existen pocas secreciones acumuladas, pero si la integridad del sistema está alterada, el inóculo que pueda llegar al parénquima pulmonar será mayor. Cuando este inóculo supera la capacidad de defensa del huésped, se produce la reacción inflamatoria cuya expresión histológica es la aparición de infiltrado agudo con leucocitos polimorfonucleares. Externamente, apreciaremos la existencia de secreciones respiratorias, que son aspiradas con sondas de aspiración por dentro del tubo endotraqueal. Se ha comprobado que una baja presión del neumotaponamiento, que permitiría un mayor paso de secreciones, se puede asociar al desarrollo de NAV, (Diaz, Lorente, Valles, \& Rello, 2010)

La clave para el desarrollo de la NAV es la presencia de un TET o traqueostomía, los cuales interfieren con la anatomía y fisiología normales del tracto respiratorio, específicamente los mecanismos funcionales envueltos en la eliminación de secreciones (tos y acción mucociliar). Los pacientes intubados tienen un nivel de conciencia reducido que compromete la eliminación voluntaria de secreciones, las cuales pueden entonces 


\section{Neumonía asociada a ventilación mecánica}

Vol. 3, núm. 3., (2019)

Andrés Alberto Vásquez Gaibor; Stefanie Cristina Reinoso Tapia; María Nora Lliguichuzca Calle; Jim Víctor Cedeño Caballero

acumularse en la orofaringe. Esto conlleva a macroaspiración y microaspiración de secreciones orofaríngeas contaminadas que son ricas en patógenos peligrosos. La flora oral normal comienza a proliferar y son capaces de viajar a lo largo del tubo endotraqueal, formando una película biológica resistente a antibióticos la cual eventualmente alcanza las vías aéreas inferiores. Los pacientes críticamente enfermos exhiben una capacidad deteriorada de suministrar una respuesta inmune a estos patógenos, conduciendo al desarrollo de una neumonía. La presencia de factores predisponentes adicionales tales como edema pulmonar en estos pacientes pueden acelerar el proceso también. La NAV de aparición temprana, que ocurre dentro de los primeros 4 días de VM, es causada usualmente por bacterias adquiridas de la comunidad sensibles a antibióticos tales como Hemofilos y Estreptococos. La NAV que se desarrolla mas de 5 días después de la iniciación de la VM es usualmente causada por bacterias resistentes a múltiples drogas tales como la Pseudomona Aeruginosa. (Miller, 2018)

\section{Factores de Riesgo}

Ballesteros, Reyes, Alarcón, \& Cervantes (2013) asegura que hay ciertos factores de riesgo que se consideran propios de la NAVM, que vienen siendo:

- Género masculino.

- Enfermedad pulmonar obstructiva crónica (EPOC).

- $\quad$ Edad mayor a los 70 años.

- Disminución del nivel de conciencia y exposición previa de antibióticos. 


\section{Neumonía asociada a ventilación mecánica}

Vol. 3, núm. 3., (2019)

Andrés Alberto Vásquez Gaibor; Stefanie Cristina Reinoso Tapia; María Nora Lliguichuzca Calle; Jim Víctor Cedeño Caballero

Sin embargo, agregan que también se encuentran otros factores de riesgo potencialmente vinculados a dicha patología, entre los que destacan:

- Uso en las primeras 48 horas de relajantes neuromusculares.

- Nutrición enteral.

- Profilaxis de úlceras de estrés.

- Traqueostomía.

- Transporte fuera de la Unidad de Cuidados Intensivos (UCI) (para, por ejemplo, un estudio de tomografía de cráneo, quirófano, entre otros).

- Drenaje de secreción subglótica.

- Descontaminación selectiva digestiva.

- Reintubación de emergencia.

- Posición prono (o decúbito ventral); que es boca abajo, tendido y con la cabeza de

lado).

- Terapia de reemplazo renal.

Los autores explican que, con una prolongada intubación orotraqueal (IOT) como con la reintubación, éstos factores podrán incrementarse significativamente. Aunque reconocen que la IOT es necesaria para la sobrevivencia de los pacientes, destacan que esta 


\section{Neumonía asociada a ventilación mecánica}

Vol. 3, núm. 3., (2019)

Andrés Alberto Vásquez Gaibor; Stefanie Cristina Reinoso Tapia; María Nora Lliguichuzca Calle; Jim Víctor Cedeño Caballero

[...] interviene con el mecanismo fisiológico normal de mantener sin contaminación bacteriana las vías respiratorias. El correcto inflado del manguito de la cánula endotraqueal $(20 \mathrm{mmHg}$ ), no está libre del deterioro del reflejo de la tos y el flujo mucociliar. La misma cánula endotraqueal drena hacia la tráquea bacterias que desarrollan neumonía. Los pacientes con IOT son más propensos a desarrollar NAVM que los pacientes traqueotomizados, porque permiten una comunicación de la tráquea con la orofaringe. La aspiración de patógenos de la orofaringe en pacientes con disfunción subglótica puede ocurrir en la extubación y reintubación. (Ballesteros, Reyes, Alarcón, \& Cervantes, 2013, pág. 79)

\section{Tratamiento}

Básicamente los tratadistas Diaz, Lorente, Valles, \& Rello (2010) dicen que:

El manejo conlleva 2 tratamientos simultáneos. Por un lado, el tratamiento de soporte y por otro, el tratamiento antibiótico. El tratamiento de soporte se inicia con una ventilación mecánica ajustada a las necesidades del paciente. De entrada, un paciente que desarrolla una NAV no está, al menos el primer día en condiciones de ser extubado. Si se estabiliza rápidamente podrá ser reevaluado. La ventilación mecánica irá dirigida a buscar la mejor oxigenación de los tejidos con el menor daño secundario. [...] Respecto al tratamiento antibiótico, lo más importante es no demorar un tratamiento efectivo ya que el tratamiento empírico inicial inadecuado conlleva una mayor mortalidad. Si la NAV es precoz y no existen estos factores de riesgo, la mayoría de las pautas empíricas presentan una cobertura correcta de la flora que nos encontraremos. Sin embargo, si el diagnóstico 


\section{Neumonía asociada a ventilación mecánica}

Vol. 3, núm. 3., (2019)

Andrés Alberto Vásquez Gaibor; Stefanie Cristina Reinoso Tapia; María Nora Lliguichuzca Calle; Jim Víctor Cedeño Caballero

de NAV se realiza en un paciente con más de una semana de hospitalización, en tratamiento antibiótico, o con factores de riesgo para multiresistentes deberemos individualizar la pauta. Si se realiza una prueba de diagnóstico etiológico y disponemos de la información de la tinción de Gram nos servirá para orientar el tratamiento empírico.

Adicionalmente, se ha encontrado un destacado e interesantísimo aporte de Torres et al. (2018), puesto que estos sintetizan la evidencia y las recomendaciones referidas como pautas internacionales en las guías clínicas de la Sociedad Europea de Enfermedades Respiratorias (European Respiratory Society o ERS, por sus siglas en inglés), la Sociedad Europea de Medicina de Cuidados Intensivos (ESICM, por sus siglas en inglés), la Sociedad Europea de Microbiología Clínica y Enfermedades Infecciosas (ESCMID, por sus siglas en inglés) e incluso la Sociedad Latinoamericana de Enfermedades Torácicas (ALAT), en cuanto al tratamiento de la neumonía adquirida en el hospital y la neumonía asociada al ventilador mecánico.

Estos tratadistas, antes de entrar en materia dejan claro que es en dos grupos en los que se clasifica la NN, es decir, la neumonía adquirida en el hospital (HAP; por sus siglas en inglés, que significan: hospital-acquired pneumonia) y la neumonía asociada a ventilador [mecánico] (VAP; por sus siglas en inglés que significan: ventilator-associated pneumonia). La HAP evoluciona posterior a las 48 horas de haber ingresado un paciente a hospitalización, y que, aunque no necesariamente necesite asistencia con ventilación artificial en el momento de su diagnóstico, pudiera ser indicada a criterio del médico tratante; mientras que la VAP, es más 


\section{Neumonía asociada a ventilación mecánica}

Vol. 3, núm. 3., (2019)

Andrés Alberto Vásquez Gaibor; Stefanie Cristina Reinoso Tapia; María Nora Lliguichuzca Calle; Jim Víctor Cedeño Caballero

propia de pacientes atendidos en la UCI que son auxiliados con ventilación mecánica, por lo menos, durante $48 \mathrm{~h}$.

De la misma manera esclarecen que dichas guías fueron "diseñadas para guiar las decisiones clínicas tomadas no solo por neumólogos e intensivistas sino por todos los profesionales de la salud que tratan a estos pacientes" están fundamentadas por evidencia (Calificación de la evaluación de recomendaciones, desarrollo y evaluación) y siguen el modelo población-intervención-comparación-resultado (PICO, por sus siglas en inglés, relativas a: population-intervention-comparison-outcome).

En específico, respecto a la VAP temprana sin factores de riesgo de resistencia a los antibióticos, han referido que son el Streptococcus pneumoniae, el Haemophilus influenzae y el Staphylococcus aureus los patógenos causantes más comunes, sensibles al tratamiento con meticilina. En la VAP tardía, contrariamente, los agentes causales tienen un alto riesgo de resistencia a los antibióticos: tales como: el Pseudomonas aeruginosa, la Acinetobacter baumannii, el Staphylococcus aureus resistente a la meticilina (MRSA, por sus siglas en inglés) y otros bacilos gramnegativos, dependiendo del microorganismo predominante en cada hospital. y UCI. Aquellos bacilos gramnegativos que son resistentes a tres o más familias de antibióticos a los que normalmente son sensibles, incluidos los betalactámicos (penicilinas y cefalosporinas), carbapenems, aminoglucósidos y quinolonas, son los que se consideran resistentes a múltiples fármacos (MDR, por sus siglas en inglés). Resaltan también que, la tasa de patógenos resistentes en los datos microbiológicos locales es alta cuando ésta supera el $25 \%$. 


\section{Neumonía asociada a ventilación mecánica}

Vol. 3, núm. 3., (2019)

Andrés Alberto Vásquez Gaibor; Stefanie Cristina Reinoso Tapia; María Nora Lliguichuzca Calle; Jim Víctor Cedeño Caballero

No obstante, previo a estas aseveraciones, han destacado que debe hacerse una diferenciación entre pacientes con factores de riesgo de resistencia a los antibióticos y pacientes sin estos factores de riesgo, e igualmente entre episodios tempranos y tardíos.

Una variable importante para identificar los patógenos MDR es el momento de aparición de la neumonía. Los HAP y VAP tempranos se definen por una ocurrencia dentro de los 4 días posteriores al ingreso al hospital y están asociados con un mejor pronóstico, ya que es más probable que sean causados por microorganismos que generalmente son sensibles a los antibióticos. Las neumonías de inicio tardío se desarrollan después de 5 días de hospitalización y es más probable que sean causadas por microorganismos MDR, con una mayor morbilidad y mortalidad. Los antibióticos de espectro estrecho pueden usarse en pacientes con inicio temprano de HAP o VAP, siempre que el riesgo de resistencia y mortalidad se considere bajo. En pacientes con HAP / VAP de inicio temprano que desarrollan shock séptico, se recomienda un tratamiento empírico de amplio espectro contra P. aeruginosa y organismos productores de BLEE. Además, MRSA debe estar cubierto si prevalece en la unidad (> 25\% de Staphylococcus aureus en la unidad). (p. 4)

Otro punto que destacan es el relacionado con el uso de uno o dos antibióticos para el tratamiento empírico inicial de amplio espectro.

Comienzan aclarando que los tratamientos antibióticos combinados se centran en:

[...] obtener sinergias entre diferentes grupos de antibióticos, extendiendo el espectro de acción contra los bacilos gramnegativos y evitando la aparición de resistencia. Sin embargo, los tratamientos combinados también pueden tener desventajas 


\section{Neumonía asociada a ventilación mecánica}

Vol. 3, núm. 3., (2019)

Andrés Alberto Vásquez Gaibor; Stefanie Cristina Reinoso Tapia; María Nora Lliguichuzca Calle; Jim Víctor Cedeño Caballero

(al menos potencialmente) como el mayor riesgo de toxicidad, el mayor costo del tratamiento y los problemas de superinfección.Las pautas europeas (y también las proporcionadas por ALAT) definen a los pacientes de alto riesgo como aquellos que presentan HAP / VAP y shock séptico y / o los siguientes factores de riesgo para patógenos MDR: 1) ingresados en unidades con altas tasas de patógenos MDR ( es decir, por encima del 25\%, incluidas las bacterias gramnegativas y MRSA); 2) uso previo de antibióticos; 3) hospitalización prolongada reciente (> 5 días); y 4) colonización previa por patógenos MDR. (p. 4)

Cuando la VAP (y HAP) es asociada a shock séptico o es producido por bacterias Gramnegativas, puede ser beneficioso el tratamiento de combinación de dos antibióticos Gramnegativos específicos. Inclusive, otros estudios revelan que la terapia dual ( $\beta$-lactamas en combinación con aminoglucósidos, fluoroquinolonas o macrólidos / clindamicina) demuestran una significativa disminución del riesgo de muerte en comparación con la monoterapia.

Es importante comenzar el tratamiento empírico combinado si existe un alto riesgo de HAP / VAP debido a bacilos gramnegativos MDR, y el paciente tiene shock séptico. Si existe un alto riesgo de MRSA en la unidad, se debe agregar un antibiótico específico dirigido a este patógeno, (p. 5)

Declaran que, actualmente, la duración del tratamiento recomendada en pacientes con VAP es de 7 a 8 días, siempre y cuando este paciente no presente otras comorbilidades respiratorias, como empiema pulmonar, absceso pulmonar, cavitación o neumonía 


\section{Neumonía asociada a ventilación mecánica}

Vol. 3, núm. 3., (2019)

Andrés Alberto Vásquez Gaibor; Stefanie Cristina Reinoso Tapia; María Nora Lliguichuzca Calle; Jim Víctor Cedeño Caballero

necrotizante, y que presenten una buena respuesta al tratamiento; esto es fines de evitar que surja algún tipo de resistencia a múltiples fármacos, sin embargo, es posible que se den algunas circunstancias determinadas en las que una terapia de mayor duración pudiera aportar igualmente beneficios.

Recomendaciones para prevención de NAVM

- Evitar la intubación orotraqueal y promover la ventilación no invasiva (VNI) siempre que sea posible.

- Realizar "vacación” de sedación en forma diaria en pacientes sin contraindicación.

- Hacer prueba de ventilación espontánea diaria en pacientes sin contraindicación.

- Cambiar los circuitos respiratorios cuando están visiblemente sucios o con disfunción.

- Higiene oral con clorhexidina solución oral $(0.12 \%)$.

- Control del paciente ventilado sin sedantes (evitar benzodiacepinas frente a cuadros de agitación).

- Movilización temprana y ejercicio en los pacientes en AVM.

- Utilizar tubos endotraqueales con aspiración subglótica para los pacientes que se espera esté en

- $\quad$ AVM más de 48 horas. 


\section{Neumonía asociada a ventilación mecánica}

Vol. 3, núm. 3., (2019)

Andrés Alberto Vásquez Gaibor; Stefanie Cristina Reinoso Tapia; María Nora Lliguichuzca

Calle; Jim Víctor Cedeño Caballero

- Realizar la esterilización o desinfección adecuada del equipo utilizado para cuidados respiratorios.

- Elevar la cabecera a $30-45^{\circ}$.

- Remover la condensación en los circuitos frecuentemente, evitando el drenaje hacia el paciente.

- Aspirar las secreciones previo a movilización.

- Mantener presión del manguito del tubo endotraqueal 20-22 cm H2O. (Cornistein, y otros, 2018)

\section{Conclusiones.}

Puede afirmarse, en base a la literatura consultada para este trabajo, que una de las causas típicas de elevadas tasas de morbimortalidad en pacientes con enfermedades críticas viene siendo precisamente la NAVM, considerándose la traqueostomía con manguito inflado (o el tubo endotraqueal) como uno de los factores de riesgo más destacado en la evolución de esta patología, por el simple hecho de que interfieren con la anatomía y fisiología propia del conducto respiratorio; no obstante, otros estudios han sumado ciertos factores igualmente trascendentes, tales como: la duración de la VM, intubación prolongada o reintubación la previa exposición a antibióticos, enfermedad pulmonar crónica, broncoaspiración, cirugía tórax/cardiotórax, presencia de dispositivos invasivos, traslados fuera de UCI para llevar a cabo otros procedimientos diagnósticos y/o terapéuticos, edad superior a los 70 años, entre otros. 


\section{Neumonía asociada a ventilación mecánica}

Vol. 3, núm. 3., (2019)

Andrés Alberto Vásquez Gaibor; Stefanie Cristina Reinoso Tapia; María Nora Lliguichuzca Calle; Jim Víctor Cedeño Caballero

Otros aspectos resaltantes de la investigación estriban en el reconocimiento que, generalmente, los especialistas refieren sobre esta complicación, pues se infiere que ésta sucede en personas que se les intuba para ser auxiliados con VM en un lapso mayor a 48 horas, existiendo por consiguiente una relación proporcionalmente directa entre la extensión del tiempo de VM y el riesgo de contagio.

Así mismo, los estudios tienden a que se asuma que aquellos casos de NAVM que se desarrollan posterior al quinto día de haber iniciado la VM, se asocia con una incrementada probabilidad de un origen bacteriológico resistente a la terapia antibiótica o farmacológica. Por ello, en perspectiva, los paquetes de prevención contra la NAVM que se adoptan en las UCI es lo que en definitiva sirve como método efectivo en la reducción de las tasas particulares de NAVM.

Finalmente, esta patología fundamenta su tratamiento o manejo en un alta sospecha clínica y, como en casi todas las enfermedades crónicas, su diagnóstico temprano e inicio oportuno del tratamiento antibiótico, adecuado a los resultados de las muestras microbiológicas, vendría siendo lo que marca la pauta en su control definitivo.

\section{Bibliografía.}

American Thoracic Society. (2017). Mechanical Ventilation. ATS, 196(1), 3-4.

Ballesteros, C., Reyes, J. M., Alarcón, L., \& Cervantes, L. (Agosto de 2013). Neumonía asociada a la ventilación mecánica. Archivos de Medicina de Urgencia de México, 5(2), 78-84. Recuperado el 10 de 09 de 2019, de https://www.medigraphic.com/pdfs/urgencia/aur2013/aur132e.pdf

Cornistein, W., Colque, Á., Staneloni, M., Lloria, M., Lares, M., González, A., . . Carbone, E. (01 de 04 de 2018). Pneumonia associated with mechanical ventilation. Update and recommendations inter- Societies SADI-SATI. Medicina, 78(2), 99-106. Recuperado el 11 de $09 \quad$ de $\quad 2019, \quad$ de 


\section{Neumonía asociada a ventilación mecánica}

Vol. 3, núm. 3., (2019)

Andrés Alberto Vásquez Gaibor; Stefanie Cristina Reinoso Tapia; María Nora Lliguichuzca Calle; Jim Víctor Cedeño Caballero

https://www.researchgate.net/publication/324571065_Pneumonia_associated_with_mech anical_ventilation_Update_and_recommendations_inter-_Societies_SADI-SATI

Diaz, E., Lorente, L., Valles, J., \& Rello, J. (Jukio de 2010). Neumonía asociada a la ventilación mecánica. Medicina Intensiva, 34(5), 318-324. Recuperado el 10 de 09 de 2019, de http://scielo.isciii.es/scielo.php?script=sci_arttext\&pid=S0210-56912010000500005

Miller, F. (27 de Junio de 2018). Worl Federation of Societies of Anaesthesiologists. Recuperado el 10 de 06 de 2019, de wfsahq.org: https://www.wfsahq.org/components/com_virtual_library/media/74d02bfd1d8ced1516fe 305f960f1698-382-Neumon--a-Asociada-a-Ventilador.pdf

Ministerio de Salud Pública del Ecuador. (09 de 06 de 2019). ENFERMEDADESRESPIRATORIAS. Recuperado el 10 de 09 de 2019, de salud.gob.ec: https://www.salud.gob.ec/wp-content/uploads/2019/09/NEUMONIA-SE-36_2019.pdf

OMS. (2019 a). Organización Mundial de la Salud. Recuperado el 10 de 09 de 2019, de who.int: https://www.who.int/gpsc/country_work/burden_hcai/es/

OMS. (2019 b). Organización Mundial de la Salud. Recuperado el 10 de 09 de 2019, de who.int/es: https://www.who.int/es/news-room/fact-sheets/detail/pneumonia

SEMI. (02 de 05 de 2019). NEUMONÍA. Recuperado el 10 de 09 de 2019, de https://www.fesemi.org/informacion-pacientes/conozca-mejor-su-enfermedad/neumonia

Torres, A., Niederman, M., Chastre, J., Ewig, S., Fernandez, P., Hanberge, H., . . . Wunderink, R. (2018). Resumen de las directrices clínicas internacionales para el tratamiento de la neumonía adquirida en el hospital y la neumonía adquirida. (E. R. Respiración], Ed.) ERJ Open Research, 4(2). doi:doi.org/10.1183/23120541.00028-2018

Vallejo, C., Ávila, S., GlendaRivera, Patiño, V., Cherrez, G., \& Cobeña, M. (2019). Manejo adecuado de ventiladores mecánicos en la UCI. RECIAMUC, 3(3), 1138-1150. doi:doi.org/10.26820/reciamuc/3.(3).julio.2019.1138-1150 